\title{
Groups with Exactly Ten Centralizers
}

\section{S. M. Jafarian Amiri ${ }^{1}$ - H. Madadi ${ }^{2}$ H. Rostami ${ }^{3}$}

Received: 10 December 2016 / Accepted: 16 October 2017 / Published online: 29 June 2018 (c) The Author(s) 2018

\section{Abstract}

In this article, we determine all groups with exactly ten element centralizers. Also we obtain the maximum size of the pairwise non-commuting elements of such groups.

Keywords Finite group · Centralizer $\cdot C A$-group

Mathematics Subject Classification Primary 20D60; Secondary 20D15

\section{Introduction}

Throughout this paper all groups mentioned are assumed to be finite and we will use usual notation; for example, $C_{n}^{j}$ and $D_{2 n}$ denote, respectively, the direct product of $j$ copies of the cyclic group of order $n$ and the dihedral group of order $2 n$. For a finite group $G, \operatorname{Cent}(G)$ denotes the set of all centralizers of single elements of $G$, i.e. $\operatorname{Cent}(G)=\left\{C_{G}(g): g \in G\right\}$, where $C_{G}(g)$ is the centralizer of the element $g$ in $G$. For a positive integer $n$, a group $G$ is called $n$-centralizer if $|\operatorname{Cent}(G)|=n$ and a primitive $n$-centralizer group if $|\operatorname{Cent}(G)|=\left|\operatorname{Cent}\left(\frac{G}{Z(G)}\right)\right|=n$ where $Z(G)$ denotes the center of $G$. Starting with Belcastro and Sherman [5], many authors have

Communicated by Hamid Mousavi.

$凶 \quad$ S. M. Jafarian Amiri

sm_jafarian@znu.ac.ir; sm.jafariana110@gmail.com

H. Madadi

halime_madadi@m-iau.ac.ir

H. Rostami

h.rostami5991@gmail.com

1 Department of Mathematics, Faculty of Sciences, University of Zanjan, Zanjan 4537138791, Iran

2 Department of Mathematics, Miyaneh branch, Islamic Azad University, Miyaneh, Iran

3 Department of Education, Molla Sadra Teaching and Training Research Center, Zanjan 4513858899, Iran 
studied the influence of $|\operatorname{Cent}(G)|$ on the structure of a group $G$ (see [1-4], [10-14] and [19-23])).

It is easy to see that $G$ is non-abelian if and only if $|\operatorname{Cent}(G)| \geq 4$. All $n$-centralizer groups have been characterized for $n \leq 9$ (see [1,2,5] and [9]). M. Zarrin [22] have generalized the previous results for infinite groups. He [19] showed that the derived length of a solvable group $G$ is $\leq|\operatorname{Cent}(G)|$. In [10] and [12] Jafarian Amiri et al. have determined all groups $G$ with $|\operatorname{Cent}(G)| \geq \frac{2}{3}|G|$. Recently, Jafarian Amiri and Rostami [13] have characterized groups having minimum number of the element centralizers among non-abelian groups of the same order.

The authors in [9] proved that the order of a 10-centralizer group is even. This result also was proved independently (in odd order case) by Foruzanfar and Mostaghim in [8], they prove that, there is no finite nonabelian group of odd order with $|\operatorname{Cent}(G)|=10$.

In this article, we determine the structure of finite groups with exactly ten centralizers. Our main result is:

Theorem 1.1 Let $G$ be a 10-centralizer group. Then $\left|\frac{G}{Z(G)}\right| \in\{16,32,56,64\}$ and $\frac{G}{Z(G)}$ has one of the following structures:

(i) $C_{2}^{4}, C_{4} \times C_{4},\left(C_{4} \times C_{2}\right) \rtimes C_{2}, D_{16}, C_{2} \times D_{8}$.

(ii) $C_{2}^{5}$ or $C_{2}^{6}$.

(iii) $C_{2}^{3} \rtimes C_{7}$, the Frobenious group of order 56 .

There are many 10-centralizer groups with the central factor of order 16 . For example by GAP [17], there exist 10-centralizer groups

$$
\begin{gathered}
G_{1}=\operatorname{SmallGroup}(128,6), G_{2}=\operatorname{SmallGroup}(128,1400) \text { and } \\
G_{3}=\operatorname{SmallGroup}(128,15)
\end{gathered}
$$

such that $\frac{G_{1}}{Z\left(G_{1}\right)} \cong C_{4} \times C_{4}, \frac{G_{2}}{Z\left(G_{2}\right)} \cong\left(C_{2}\right)^{4}$ and $\frac{G_{3}}{Z\left(G_{3}\right)} \cong\left(C_{4} \times C_{2}\right) \rtimes C_{2}$.

In general, the converse of Theorem 1.1 is not true. For example by GAP [17], it can be checked that SmallGroup $(32,50)$ has sixteen centralizers while its central factor group is isomorphic to $C_{2}^{4}$ and $\operatorname{SmallGroup}(64,14)$ is 12-centralizer while its central factor group is isomorphic to $\left(C_{4} \times C_{2}\right) \rtimes C_{2}$. Also the central factor group of SmallGroup $(64,132)$ is isomorphic to $C_{2} \times D_{8}$ whereas this group is 12-centralizer, the central factor group of $\operatorname{SmallGroup}(128,2216)$ is isomorphic to $C_{2}^{5}$ whereas this group is 27-centralizer and $G=\operatorname{SmallGroup}(128,2326)$ is 64-centrlizer group with $\frac{G}{Z(G)} \cong C_{2}^{6}$. But in Proposition 4.2 we will show that converse of Theorem 1.1 is true whenever $\frac{G}{Z(G)} \cong C_{4} \times C_{4}$ or $D_{16}$. Also in Proposition 4.3, it is shown that if $\frac{G}{Z(G)} \cong C_{2}^{3} \rtimes C_{7}$, then $|\operatorname{Cent}(G)| \in\{10,14,16\}$. However, we could not find a group $G$ such that $\frac{G}{Z(G)} \cong C_{2}^{3} \rtimes C_{7}$ and $\mid \operatorname{Cent}(G \mid=14$ or 16 .

The following result is an immediate consequence of Theorem 1.1.

Corollary 1.2 If $G$ is a primitive 10-centralizer group, then $\frac{G}{Z(G)} \cong C_{2}^{3} \rtimes C_{7}$. 


\section{Definitions, Notations and Preliminaries}

There are interesting relations between centralizers and pairwise non-commuting elements in groups (see [1, Proposition 2.5 and Lemma 2.6]). Let $G$ be a finite non-abelian group and let $X$ be a subset of pairwise non-commuting elements of $G$ such that $|X| \geq|Y|$ for any other set of pairwise non-commuting elements $Y$ in $G$. Then the subset $X$ is said to have the maximum size, and this size is denoted by $\omega(G)$. Also $\omega(G)$ is the maximum clique size in the non-commuting graph of a finite group $G$. The non-commuting graph of a group $G$ is defined as a graph whose $G \backslash Z(G)$ is the set of vertices and two vertices are joined if and only if they do not commute. By a famous result of Neumann [15] answering a question of Erdös, the finiteness of $\omega(G)$ is equivalent to the finiteness of the factor group $\frac{G}{Z(G)}$ which follows that $|\operatorname{Cent}(G)|$ is finite. In addition, if $G$ has a finite number of centralizers, then it is easy to see that $\omega(G)$ is finite.

To prove the main theorem, we need the following results.

Remark 2.1 Let $G$ be a group and $\left\{x_{1}, \ldots, x_{\omega(G)}\right\}$ be a set of pairwise non-commuting elements of $G$ such that $\left|G: C_{G}\left(x_{i}\right)\right|=\alpha_{i}$ with $\alpha_{1} \leq \alpha_{2} \leq \cdots \leq \alpha_{\omega(G)}$. Then we have the following.

(i) $G=\bigcup_{i=1}^{\omega(G)} C_{G}\left(x_{i}\right)$ and $Z(G)=\bigcap_{i=1}^{\omega(G)} C_{G}\left(x_{i}\right)$ (see Theorem 5.1 of [18]).

(ii) $\alpha_{2} \leq \omega(G)-1$ (see [18, Lemma 3.3]).

(iii) If $\alpha_{2}=\omega(G)-1$, then $\alpha_{i}=\omega(G)-1$ for each $i>1$ and $C_{G}\left(x_{i}\right) \cap C_{G}\left(x_{j}\right) \leq$ $C_{G}\left(x_{1}\right)$ for every $i \neq j$ (see [18, Lemma 3.3]). In addition, it is easy to see that $\left|\frac{G}{Z(G)}\right|=\sum_{i=2}^{\omega(G)}\left|\frac{C_{G}\left(x_{i}\right)}{Z(G)}\right|$.

Lemma 2.2 Let $G$ be a finite group and $k \leq 6$ be a positive integer. Then, $k$ is the number of non-abelian centralizers belonging to $\operatorname{Cent}(G)$ if and only if $|\operatorname{Cent}(G)|=$ $\omega(G)+k$.

Proof See [11, Theorem 1.2].

Lemma 2.3 Let $G$ be finite group and $x, y \in G$ such that $x y \neq y x$. If $C_{G}(x)$ is abelian of prime index $p$ in $G$, then $C_{G}(x) \cap C_{G}(y)=Z(G)$ and $\left|\frac{G}{Z(G)}\right| \leq p\left|G: C_{G}(y)\right|$.

Proof The proof is straightforward.

Let $G$ be a non-trivial group. A collection $\Pi$ of non-trivial subgroups of $G$ is said to be a partition of $G$ if every non-trivial element of $G$ belongs to a unique subgroup in $\Pi$. Recall that a group $G$ is a $C A$-group $(G \in C A)$ if $C_{G}(x)$ is abelian for every $x \in G \backslash Z(G)$. It is easy to see that if $G$ is a $C A$-group, then $\left\{\frac{C_{G}(g)}{Z(G)}: g \in G \backslash Z(G)\right\}$ is a partition of $\frac{G}{Z(G)}$. By [1, Lemma 2.6], $G$ is a $C A$-group if and only if $|\operatorname{Cent}(G)|=$ $\omega(G)+1$.

\section{Some Special Cases of 10-Centralizer Groups}

In this section, we prove some results that we will need in the sequel. Suppose that $G$ is a 10-centralizer group. By [1, Lemma 2.4], we have $5 \leq \omega(G) \leq 9$. In particular, 
$\omega(G)=9$ if and only if $G$ is a $C A$-group. We first prove that if $\omega(G) \neq 9$, then $\frac{G}{Z(G)}$ is of order 16.

Lemma 3.1 Suppose that $G$ is a 10-centralizer group. If $\omega(G) \in\{5,6,7,8\}$, then $\left|\frac{G}{Z(G)}\right|=16$.

Proof If $\omega(G)=5$, then $G$ is not a $C A$-group and so $\left|\frac{G}{Z(G)}\right|$ has at least four (not necessarily distinct) prime divisors by [4, Lemma 2.1]. In addition, we have $\left|\frac{G}{Z(G)}\right| \leq$ 16 by $[1$, Remark $2.1(3)]$. Therefore, $\left|\frac{G}{Z(G)}\right|=16$, as wanted.

Suppose that $\omega(G)=6$ and $\left\{x_{1}, \ldots, x_{6}\right\}$ is a set of pairwise non-commuting elements of $G$ such that $\left|G: C_{G}\left(x_{i}\right)\right|=\alpha_{i}$ with $\alpha_{1} \leq \alpha_{2} \leq \cdots \leq \alpha_{6}$. By Lemma 2.2, $C_{G}\left(x_{i}\right)$ is abelian for each $i$ and so $G$ has three proper non-abelian centralizers, say $C_{G}(a), C_{G}(b), C_{G}(c)$. Also $G$ is not a $C A$-group which implies that $\left|\frac{G}{Z(G)}\right|$ has at least four (not necessarily distinct) prime divisors. Therefore, $\left|\frac{G}{Z(G)}\right| \geq 16$. We have to prove that $\left|\frac{G}{Z(G)}\right|=16$.

Suppose, for a contradiction, that $\left|\frac{G}{Z(G)}\right|>16$. Since $\omega(G)=6$, we have $\alpha_{2} \leq 5$ by Remark 2.1(ii). If $\alpha_{2}=5$, then by Lemma 2.3, $\left|\frac{G}{Z(G)}\right| \in\{20,25\}$ which follows that $G$ is a $C A$-group, a contradiction. Hence $\alpha_{2}=4$. Similarly $\alpha_{1}=4$. Since $\left|\frac{G}{Z(G)}\right|>16$ and $C_{G}\left(x_{i}\right)$ is abelian for $i \in\{1,2\}$, we have $C_{G}\left(x_{1}\right) \cup C_{G}\left(x_{2}\right) \subseteq C_{G}(g)$ for some $g \in\left(C_{G}\left(x_{1}\right) \cap C_{G}\left(x_{2}\right)\right) \backslash Z(G)$ and so $G=C_{G}(g) \cup\left(\cup_{i=3}^{6} C_{G}\left(x_{i}\right)\right.$. Therefore, $\alpha_{3} \leq 4$ by $\left[18\right.$, Lemma 3.3] and hence $\alpha_{1}=\cdots=\alpha_{6}=4$. Consequently $C_{G}\left(x_{i}\right) \cap C_{G}\left(x_{j}\right) \neq$ $Z(G)$ and $C_{G}\left(x_{i}\right) \cap C_{G}\left(x_{j}\right) \cap C_{G}\left(x_{k}\right)=Z(G)$ for every distinct $i, j, k$. Also by indices $\alpha_{i}^{\prime}$ s and considering the covering of $G$ by its centralizers, we conclude that each of the non-abelian centralizers $C_{G}(a), C_{G}(b), C_{G}(c)$ contains exactly two distinct centralizers $C_{G}\left(x_{i}\right)$ and $C_{G}\left(x_{j}\right)$ for some $i \neq j$, say $C_{G}\left(x_{1}\right) \cup C_{G}\left(x_{2}\right) \subseteq C_{G}(a)$, $C_{G}\left(x_{3}\right) \cup C_{G}\left(x_{4}\right) \subseteq C_{G}(b)$ and $C_{G}\left(x_{5}\right) \cup C_{G}\left(x_{6}\right) \subseteq C_{G}(c)$. On the other hand, we have $C_{G}\left(x_{1}\right) \cap C_{G}\left(x_{3}\right) \neq Z(G)$ which implies that $C_{G}\left(x_{1}\right) \cup C_{G}\left(x_{3}\right)$ are contained in one of $C_{G}(a), C_{G}(b)$ or $C_{G}(c)$. Therefore, one of the non-abelian centralizers contains at least three centralizers $C_{G}\left(x_{i}\right)^{\prime} s$, a contradiction. This completes the proof of this case.

The proof is similar when $\omega(G)=7$ or 8 .

In view of $[1$, Lemmas 3.3] and $[9,5.6]$, it is necessary to investigate all 10centralizer groups $G$ with $\left|\frac{G}{Z(G)}\right|=24$ or 36 , separately.

Lemma 3.2 If $G$ is a 10-centralizer group, then $\left|\frac{G}{Z(G)}\right| \neq 24$ or 36 .

Proof Let $\left\{x_{1}, \ldots, x_{\omega(G)}\right\}$ is a set of pairwise non-commuting elements of $G$ such that $\left|G: C_{G}\left(x_{i}\right)\right|=\alpha_{i}$ with $\alpha_{1} \leq \alpha_{2} \leq \cdots \leq \alpha_{\omega(G)}$. Put $\bar{G}=\frac{G}{Z(G)}$.

Suppose, for a contradiction, that $|\bar{G}|=24$. It follows from Lemma 3.1 that $\omega(G)=$ 9. Therefore, $G$ is a $C A$-group and $\alpha_{2} \leq 8$ by Remark 2.1(ii). Also $\Gamma=\left\{\frac{C_{G}\left(x_{i}\right)}{Z(G)}: 1 \leq\right.$ $i \leq 9\}$ is a partition of $\bar{G}$. If $\alpha_{2}=8$, then $\alpha_{2}=\alpha_{3}=\cdots=\alpha_{9}=8$ by Remark 2.1(iii). Therefore, the number of the Sylow three-subgroups of $\bar{G}$ is eight, a contradiction. Since $C_{G}\left(x_{1}\right) \cap C_{G}\left(x_{2}\right)=Z(G)$, we have $\alpha_{2}=6$ and so $\alpha_{1}=4$ or 6 . It follows from [7, Theorem A] that either $G$ has an abelian normal subgroup of prime index or $\bar{G}$ is a 
Frobenius group with an abelian complement. By the order of $\bar{G}$ and indices $\alpha_{i}$ 's, the first case cannot be happened. Hence, $\bar{G}$ is Frobenius. If $\alpha_{1}=4$, then $\frac{C_{G}\left(x_{1}\right)}{Z(G)}$ is a normal cyclic subgroup of $\bar{G}$ of order six and so the center of $\bar{G}$ is nontrivial, a contradiction. Therefore, $\alpha_{1}=\alpha_{2}=6$. By partition $\Gamma$ and the order of $\bar{G}$, we see that $\frac{G}{Z(G)}$ has three Sylow two-subgroups. If the number of Sylow three-subgroups of $\frac{G}{Z(G)}$ is greater than one, then $\frac{G}{Z(G)} \cong S_{4}$ which implies that $11=\omega(\bar{G}) \leq \omega(G)$, a contradiction. Thus, $\frac{G}{Z(G)}$ has a unique Sylow three-subgroup $\bar{Q}$ and so this is the Frobenius kernel of $\bar{G}$ since the orders of the kernel and complement of a Frobenius group is relatively prime. If $\frac{H}{Z(G)}$ is the Frobenius complement, then by [7, Theorem A], $H$ is abelian which follows $|\operatorname{Cent}(G)| \neq 10$ by [9, Proposition 3.1] and this is not possible.

Now suppose, for a contradiction, that $|\bar{G}|=36$. Similarly, we have $\omega(G)=9$ and so the result gets from [9, Corollary 3.2].

We shall investigate the structure of 10-centralizer groups $G$ such that $\frac{G}{Z(G)}$ is a two-group and this is one of the most important special cases (see [1, Lemma 3.2], [2, Corollary 3.3] and [9, Lemmas 4.2-4.6]). Suppose that $G$ is a 10-centralizer group such that $\left|\frac{G}{Z(G)}\right|=2^{k}$ for some positive integer $k$. If $\omega(G) \in\{5,6,7,8\}$, then $\left|\frac{G}{Z(G)}\right|=16$ by Lemma 3.1. If $\omega(G)=9$, then $\alpha_{2} \leq 8$ and $G$ is a $C A$-group. It follows that $C_{G}\left(x_{1}\right) \cap C_{G}\left(x_{2}\right)=Z(G)$ and so $\left|\frac{G}{Z(G)}\right| \leq\left|G: C_{G}\left(x_{1}\right)\right|\left|G: C_{G}\left(x_{2}\right)\right| \leq 64$. Therefore, we have the following two results.

Lemma 3.3 If $G$ is a 10-centralizer group such that $\left|\frac{G}{Z(G)}\right|=32$, then $\frac{G}{Z(G)} \cong C_{2}^{5}$.

Proof It follows from Lemma 3.1 that $\omega(G)=9$. Consequently $G$ is a $C A$-group. If $\left\{x_{1}, \ldots, x_{9}\right\}$ is a set of pairwise non-commuting elements of $G$ such that $\mid G$ : $C_{G}\left(x_{i}\right) \mid=\alpha_{i}$ with $\alpha_{1} \leq \alpha_{2} \leq \cdots \leq \alpha_{9}$, then $\Gamma=\left\{\frac{C_{G}\left(x_{i}\right)}{Z(G)}: 1 \leq i \leq 9\right\}$ is a partition of $\bar{G}, \alpha_{1}=4$ and $\alpha_{2}=\cdots=\alpha_{9}=8$. Therefore, the class sizes in $G$ are $\{1,4,8\}$ and so $\exp \left(\frac{G}{Z(G)}\right)=2$ by [7, Lemma 6.1]. This completes the proof.

Lemma 3.4 If $G$ is a 10-centralizer group such that $\left|\frac{G}{Z(G)}\right|=64$, then $\frac{G}{Z(G)} \cong C_{2}^{6}$.

Proof The proof is similar to Lemma 3.3.

\section{Proofs of the Main Results}

In what follows we determine all 10-centralizer groups $G$ such that $\frac{G}{Z(G)}$ is not a two-group.

Proposition 4.1 Let $G$ be a 10-centralizer group. If $\frac{G}{Z(G)}$ is not a two-group, then $\frac{G}{Z(G)} \cong C_{2}^{3} \rtimes C_{7}$.

Proof Suppose that $\left\{x_{1}, \ldots, x_{\omega(G)}\right\}$ is a set of pairwise non-commuting elements of $G$ such that $\left|G: C_{G}\left(x_{i}\right)\right|=\alpha_{i}$ with $\alpha_{1} \leq \alpha_{2} \leq \cdots \leq \alpha_{\omega(G)}$. It follows from Lemma 3.1 that $\omega(G)=9$. Therefore, $G$ is a $C A$-group and by Remark 2.1(ii), $\alpha_{2} \leq 8$ which implies that $\left|\frac{G}{Z(G)}\right| \leq \alpha_{1} \alpha_{2} \leq 64$. By [20, Theorem A], $G$ is solvable. Since $|G|$ is 
even by [9, Proposition 5.8], we have either $\frac{G}{Z(G)}$ is Frobenius or $G$ has an abelian normal subgroup of prime index by [7, Theorem A]. We claim that $\left|\frac{G}{Z(G)}\right|=56$.

If $G$ has an abelian normal subgroup of prime index $p$, then $\left|G^{\prime}\right|=8$ by [4, Theorem 2.3] and so $\left|\frac{G}{Z(G)}\right|=8 p$. Therefore, $p=5$ or seven by Lemma 3.2. If $p=5$, then $\left|\frac{G}{Z(G)}\right|=40$ and $\alpha_{2}=8$. It follows that $\alpha_{i}=8$ for each $i>1$ and hence $\frac{G}{Z(G)}$ has at least eight Sylow five-subgroups which is a contradiction. Thus, $p=7$ and the claim is proved in this case. So we may assume that $G$ does not contain any normal abelian subgroup of prime index. Therefore, $\frac{G}{Z(G)}$ is Frobenius. Now, we want to show that $\alpha_{2}=8$.

It follows from [1, Proposition 2.5 (a)(2)] that $\alpha_{2} \geq 4$. Clearly $\alpha_{2} \neq 5$. If $\alpha_{2}=4$ or six, then $\left|\frac{G}{Z(G)}\right| \leq 36$ and so $\left|\frac{G}{Z(G)}\right| \in\{12,18,30\}$ by Lemma 3.2. If $\left|\frac{G}{Z(G)}\right|=12$, then $\frac{G}{Z(G)} \cong A_{4}$ and so by [2, Corollary 3.6], we have $|\operatorname{Cent}(G)|=6$ or eight which is impossible. If $\left|\frac{G}{Z(G)}\right|=30$, then $G$ has an abelian normal subgroup of index two, a contradiction. If $\left|\frac{G}{Z(G)}\right|=18$, then a Sylow three-subgroup of $\frac{G}{Z(G)}$ is the Frobenius kernel of order nine and so $|\operatorname{Cent}(G)| \geq 11$ by [9, Proposition 3.1], a contradiction. Let $\alpha_{2}=7$. Then $\left|\frac{G}{Z(G)}\right| \leq 49$. By [4, Proposition 2.9], we have $\left|\frac{G}{Z(G)}\right|=28$ or 42. Since there is no Froubenius group of order $28,\left|\frac{G}{Z(G)}\right|=42$. But by [9, Proposition 3.1], we have $|\operatorname{Cent}(G)|=9$ or greater than 10 which is impossible. Therefore, $\alpha_{2}=8$. It follows from Remark 2.1 (ii) that $\alpha_{i}=8$ for $i>1$ and $\left|\frac{G}{Z(G)}\right|=56$. This complete the proof of the claim.

Since $\alpha_{i}=8$ for $i \geq 2$ and $\alpha_{1}=7$, we conclude that $\frac{G}{Z(G)}$ is a Frobenius group. But among group of order 56, only $C_{2}^{3} \rtimes C_{7}$ has trivial center. This completes the proof.

The group $G$ is said to be capable if there exists a group $H$ such that $G \cong \frac{H}{Z(H)}$. The epicenter of $G$, denoted by $Z^{\star}(G)$, is the smallest normal subgroup of $G$ such that $\frac{G}{Z^{\star}(G)}$ is capable. In [6], it is proved that a group $G$ is capable if and only if $Z^{\star}(G)=1$. Now, we are ready to prove the main result.

Proof of Theorem 1.1 Assume that $\left\{x_{1}, \ldots, x_{\omega(G)}\right\}$ is a set of pairwise non-commuting elements of $G$ such that $\left|G: C_{G}\left(x_{i}\right)\right|=\alpha_{i}$ with $\alpha_{1} \leq \alpha_{2} \leq \cdots \leq \alpha_{\omega(G)}$. Then $5 \leq \omega(G) \leq 9$ by [1, Lemma 2.4] and $\alpha_{2} \leq \omega(G)-1$ by Remark 2.1(ii).

We first assume that $\frac{G}{Z(G)}$ is a two-group. If $\omega(G) \neq 9$, then $\left|\frac{G}{Z(G)}\right|=16$ by Lemma 3.1. But it is easily checked by GAP [17] that the only capable groups of order 16 are $C_{4} \times C_{4},\left(C_{4} \times C_{2}\right) \rtimes C_{2}, C_{2} \times D_{8}, D_{16},\left(C_{2}\right)^{4}$, as desired. Now we may assume that $\left|\frac{G}{Z(G)}\right|>16$. Then $\omega(G)=9$ and so $G$ is a $C A$-group which implies $\left|\frac{G}{Z(G)}\right| \leq \alpha_{1} \alpha_{2} \leq 64$. Thus, $\left|\frac{G}{Z(G)}\right|=32$ or 64 . We conclude that $\frac{G}{Z(G)} \cong C_{2}^{5}$ or $C_{2}^{6}$ by Lemmas 3.3 and 3.4, respectively.

Next, suppose that $\frac{G}{Z(G)}$ is not a two-group. Then $\frac{G}{Z(G)} \cong\left(C_{2}\right)^{3} \rtimes C_{7}$ by Proposition 4.3. This completes the proof.

In the following result, we compute the number of centralizers for all groups with central factor isomorphic to $C_{4} \times C_{4}$ or $D_{16}$. So in these cases convers of Theorem 1.1 is hold. 
Proposition 4.2 Let $G$ be a non-abelian group such that $\frac{G}{Z(G)} \cong C_{4} \times C_{4}$ or $D_{16}$. Then $|\operatorname{Cent}(G)|=10$.

Proof Suppose that $\bar{G}=\frac{G}{Z(G)} \cong C_{4} \times C_{4}$ and $\left\{x_{1}, \ldots, x_{\omega(G)}\right\}$ is a set of pairwise non-commuting element of $G$. Since $C_{4} \times C_{4}$ has six cyclic subgroups of order four and $\bar{G}=\cup_{i=1}^{\omega(G)} \frac{C_{G}\left(x_{i}\right)}{Z(G)}$, we have $\omega(G) \leq 6$. But $\omega(G) \geq 5$ by [9, Remark 2.4]. By [14, Theorem 1.3], $\omega(G) \neq 5$ and so $\omega(G)=6$. It follows that $\frac{C_{G}\left(x_{i}\right)}{Z(G)}=\left\langle x_{i} Z(G)\right\rangle$ is of order four. Since $\bar{G}$ has only three elements of order two, we may assume that $\frac{C_{G}\left(x_{1}\right)}{Z(G)} \cap \frac{C_{G}\left(x_{2}\right)}{Z(G)}=\left\langle x_{1}^{2} Z(G)\right\rangle, \frac{C_{G}\left(x_{3}\right)}{Z(G)} \cap \frac{C_{G}\left(x_{4}\right)}{Z(G)}=\left\langle x_{3}^{2} Z(G)\right\rangle$ and $\frac{C_{G}\left(x_{5}\right)}{Z(G)} \cap \frac{C_{G}\left(x_{6}\right)}{Z(G)}=$ $\left\langle x_{5}^{2} Z(G)\right\rangle$. Since $C_{G}\left(x_{i}\right) \subset C_{G}\left(x_{i}^{2}\right) \subset G$ for $i \in\{1,3,5\}$. Therefore,

$$
\operatorname{Cent}(G)=\left\{G, C_{G}\left(x_{i}\right): 1 \leq i \leq 6\right\} \bigcup\left\{C_{G}\left(x_{i}^{2}\right): i=1,3,5\right\}
$$

which implies that $|\operatorname{Cent}(G)|=10$.

Now, let $\frac{G}{Z(G)} \cong D_{16}$. It follow from [1, Proposition 2.2] that $|\operatorname{Cent}(G)|=10$ and the proof is complete.

Now, we compute $|\operatorname{Cent}(G)|$ for any group $G$ with $\frac{G}{Z(G)} \cong C_{2}^{3} \rtimes C_{7}$.

Proposition 4.3 Let $G$ be finite group. If $\frac{G}{Z(G)} \cong C_{2}^{3} \rtimes C_{7}$, then $|\operatorname{Cent}(G)| \in$ $\{10,14,16\}$.

Proof Since $\frac{G}{Z(G)}$ is a Frobenius group with the abelian Frobenius kernel and abelian Frobenius complement, $G$ is a $C A$-group by [7, Theorem A] (see also [16]). Let $\frac{K}{Z(G)}$ be the Frobenius kernel of $\frac{G}{Z(G)}$. So by [16, Lemma 5.5], we consider two following cases:

Case 1. If $Z(G) \neq Z(K)$, then $|\operatorname{Cent}(G)|=|K: Z(G)|+2=10$.

Case 2. If $Z(G)=Z(K)$, then $|\operatorname{Cent}(G)|=|K: Z(G)|+|\operatorname{Cent}(K)|$. Since $\frac{K}{Z(K)} \cong C_{2}^{3}$, [1, Proposition 3.4] yields that $|\operatorname{Cent}(K)|=6$ or 8 . Therefore, $|\operatorname{Cent}(G)|=14$ or 16 and we are done.

Proof of Corollary 1.2 Since $C_{2}^{3} \rtimes C_{7}$ is a 10-centralizer group and the remainder groups listed in the Theorem 1.1 are not 10-centralizer, we have the result.

Acknowledgements The authors are indebted to the referee for his/her careful reading and valuable comments.

Open Access This article is distributed under the terms of the Creative Commons Attribution 4.0 International License (http://creativecommons.org/licenses/by/4.0/), which permits unrestricted use, distribution, and reproduction in any medium, provided you give appropriate credit to the original author(s) and the source, provide a link to the Creative Commons license, and indicate if changes were made. 


\section{References}

1. Abdollahi, A., Jafarian Amiri, S.M., Hassanabadi, A.M.: Groups with specific number of centralizers. Houston J. Math. 33(1), 43-57 (2007)

2. Ashrafi, A.R.: Counting the centralizers of some finite groups. Korean J. Comput. Appl. Math 7(1), 115-124 (2000)

3. Ashrafi, A., Taeri, B.: On finite groups with a certain number of centralizers. J. Appl. Math. Comput. 7, 217-227 (2005)

4. Baishya, S.J.: On finite groups with specific number of centralizers. Int. Electron. J. Algebra 13, 53-62 (2013)

5. Belcastro, S.M., Sherman, G.J.: Counting centralizers in finite groups. Math. Mag. 5, 111-114 (1994)

6. Beyl, F.R., Felgner, U., Schmid, P.: On groups occurring as center factor groups. J. Algebra 61(1), 161-177 (1979)

7. Dolfi, S., Herzog, M., Jabara, E.: Finite groups whose noncentral commuting elements have centralizers of equal size. Bull. Aust. Math Soc. 82, 293-304 (2010)

8. Foruzanfar, Z., Mostaghim, Z.: On 10-centralizer groups of odd order. ISRN Algebra 2014, 4 (2014). (Article ID 607984)

9. Jafarian Amiri, S.M., Madadi, H., Rostami, H.: On 9-centralizer groups. J. Algebra Appl 14(1), 1550003 (2015)

10. Jafarian Amiri, S.M., Madadi, H., Rostami, H.: Finite groups have even more centralizers. Bull. Iran. Math. Soc. 41(6), 1423-1431 (2015)

11. Jafarian Amiri, S.M., Rostami, H.: Groups with a few non-abelian centralizers. Publ. Math. Debrecen 87(3-4), 429-437 (2015)

12. Jafarian Amiri, S.M., Amiri, M., Rostami, H.: Finite groups determined by the number of element centralizers. Comm. Algebra. 45(9), 3792-3797 (2017)

13. Jafarian Amiri, S.M., Rostami, H.: Centralizers and the maximum size of the pairwise non-commuting elements in finite groups. Hacettepe J. Math. 46(2), 193-198 (2017)

14. Jafarian Amiri, S.M., Amiri, H., Madadi, H.: On the maximum number of the pairwise non-commuting elements in a finite group. J. Algebra Apply 16(1), 1650197 (2017). (9 pages)

15. Neumann, B.H.: A problem of Paul Erdos on groups. J. Aust. Math. Soc. 21(21), 467-472 (1976)

16. Schmidt, R.: Zentralisatorverbände endlicher gruppen. Rend. Sem. Mat. Univ. Padova 44, 97-131 (1970)

17. The GAP Team, GAP-Groups: Algoritms, and programming, version 4.5. 7 (2012). http://www.gapsystem.org. Accessed 13 Feb 2015

18. Tomkinson, M.J.: Groups covered by finitely many cosets or subgroups. Comm. Algebra 15, 845-859 (1987)

19. Zarrin, M.: Criteria for the solubility of finite groups by its centralizers. Arch. Math. 96, 225-226 (2011)

20. Zarrin, M.: On element centralizers in finite groups. Arch. Math. 93, 497-503 (2009)

21. Zarrin, M.: On solubility of groups with finitely many centralizers. Bull. Iran. Math. Soc. 39, 517-521 (2013)

22. Zarrin, M.: On non-commuting sets and centralisers in infinite groups. Bull. Aust. Math. Soc. 93(1), 42-46 (2016)

23. Zarrin, M.: On noncommuting sets and centralizers in finite groups. Bull. Aust. Math. Soc. 10, 1-5 (2015) 